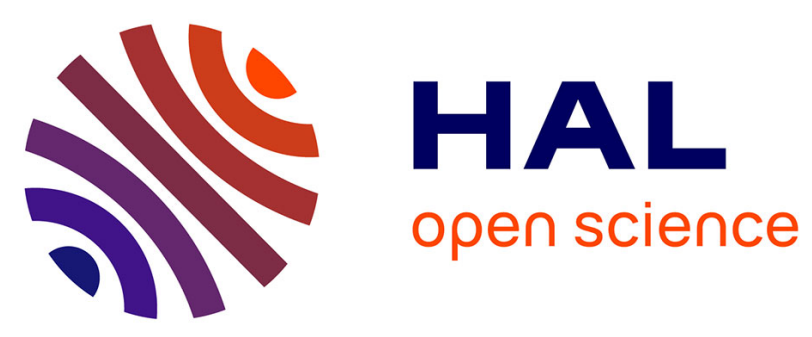

\title{
A 300-GHz wireless link employing a photonic transmitter and an active electronic receiver with a transmission bandwidth of $54 \mathrm{GHz}$
}

Iulia Dan, Vinay Chinni, Pascal Szriftgiser, Emilien Peytavit, Jean-Francois

Lampin, Malek Zegaoui, Mohammed Zaknoune, Guillaume Ducournau, Ingmar Kallfass

\section{To cite this version:}

Iulia Dan, Vinay Chinni, Pascal Szriftgiser, Emilien Peytavit, Jean-Francois Lampin, et al.. A 300-GHz wireless link employing a photonic transmitter and an active electronic receiver with a transmission bandwidth of $54 \mathrm{GHz}$. IEEE Transactions on Terahertz Science and Technology, 2020, 10, pp.271-281. 10.1109/tthz.2020.2977331 . hal-02997786

\section{HAL Id: hal-02997786 \\ https://hal.science/hal-02997786}

Submitted on 26 Nov 2020

HAL is a multi-disciplinary open access archive for the deposit and dissemination of scientific research documents, whether they are published or not. The documents may come from teaching and research institutions in France or abroad, or from public or private research centers.
L'archive ouverte pluridisciplinaire HAL, est destinée au dépôt et à la diffusion de documents scientifiques de niveau recherche, publiés ou non, émanant des établissements d'enseignement et de recherche français ou étrangers, des laboratoires publics ou privés. 


\title{
$300 \mathrm{GHz}$ Wireless Link Employing a Photonic Transmitter and an Active Electronic Receiver with a Transmission Bandwidth of $54 \mathrm{GHz}$
}

\author{
Iulia Dan, Vinay Chinni, Pascal Szriftgiser, Emilien Peytavit, Jean-François Lampin, Malek Zegaoui, Mohammed \\ Zaknoune, Guillaume Ducournau, and Ingmar Kallfass
}

\begin{abstract}
In this paper, we present a $300 \mathrm{GHz}$ wireless link composed of a photonic uni-traveling-carrier diode transmitter and an active electronic receiver based on millimeterwave integrated circuits fabricated in an InGaAs metamorphic high electron mobility transistor technology. The input pseudo-random binary sequence is transmitted and analyzed offline using fast analog to digital converters. The data transmission reaches $100 \mathrm{Gbps}$ over a distance of $15 \mathrm{~m}$. Complex modulation formats, like 32-Quadrature Amplitude Modulation and 64-Quadrature Amplitude Modulation, are successfully transmitted up to a symbol rate of $8 \mathrm{GBd}$. The system presents not only high linearity but is also capable of transmitting high symbol rates, up to 40 GBd. To the best of the authors' knowledge, this represents the highest ever reported transmission bandwidth as well as the highest spectral density symbol rate product for transmissions with center frequency in the terahertz band. Thus, the usage of such links in future bandwidth-hungry applications like data centers, data showers, front- and backhaul is proven feasible.
\end{abstract}

Index Terms-Complex modulation, millimeter wave monolithic integrated circuits, Photomixers, radio link, terahertz (THz) communications, wireless communication.

\section{INTRODUCTION}

Transmitters and receivers, or, when combined into an integrated functional sub-system, so-called "transceivers" (TRX), are at the heart of any wireless communication system. They combine the Terahertz $(\mathrm{THz})$ frequency range as a channel with signal sourcing and sinking through the digital signal processing (DSP) domain in the baseband. Since no direct DSP is yet possible at $\mathrm{THz}$ frequencies, signals need to be frequencyshifted into and from the $\mathrm{THz}$ range. This frequency-shifting is performed by the TRX, which can be implemented based either on fully electronic, fully photonic or mixed electronicphotonic technologies. The latter approach is today the most promising way forward since it allows to combine the unique advantages of both domains, namely the low receiver noise, high transmit power and high functional integration density of electronics-based $\mathrm{THz} \mathrm{TRX}$, with the high spectral purity and

Iulia Dan and Ingmar Kallfass are with the Institute of Robust Power Semiconductor Systems, University of Stuttgart, Stuttgart, Germany e-mail: iulia.dan@ilh.uni-stuttgart.de.

Pascal Szriftgiser is with the Laboratoire de Physique des Lasers Atomes et Molécules (PhLAM), UMR CNRS 8523 / Université de Lille, 59652 Villeneuve d'Asq cedex, France.

Vinay Chinni, Emilien Peytavit, Jean-François Lampin, Malek Zegaoui, Mohammed Zaknoune and Guillaume Ducournau are with the Institut d'Electronique, Microélectronique et de Nanotechnologie (IEMN), UMR CNRS 8520 / Université de Lille, 59652 Villeneuve d'Asq cedex, France.

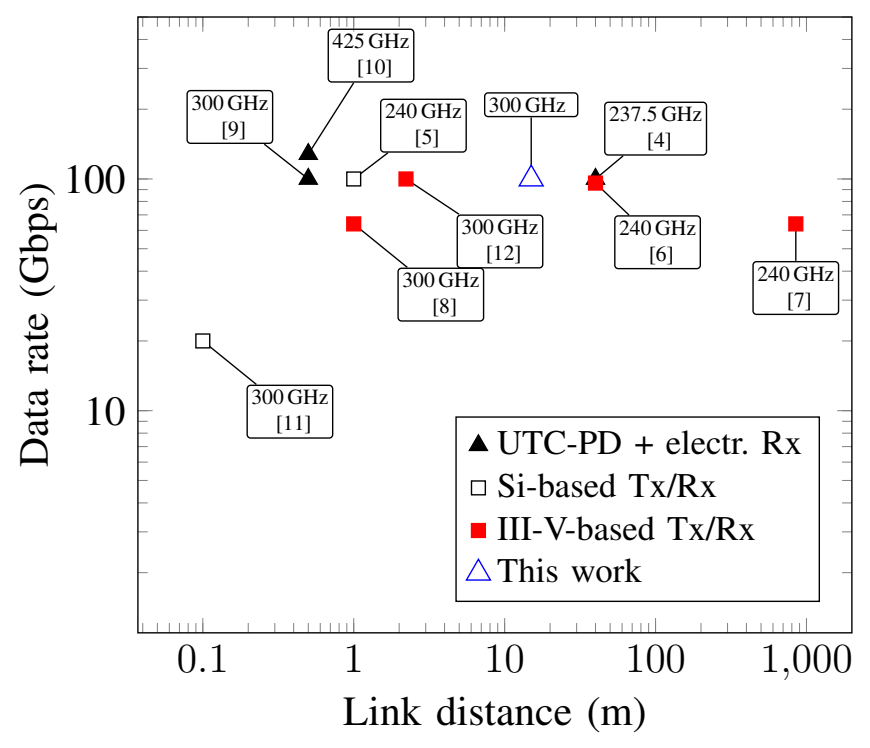

Fig. 1: State of the art in millimeterwave wireless communication links operating at carrier frequencies above $100 \mathrm{GHz}$. Experiments where the $\mathrm{mmW}$ signal is generated in the optical domain, using uni-traveling-carrier photo diodes (UTC-PD) are indicated by triangles. MMIC based measurements are indicated by squares.

high quality phase and frequency control of photonics-based TRX [1], [2].

The need for higher wireless data rates and the fact that the frequency spectrum beyond $257 \mathrm{GHz}$, where huge bandwidths are available, has recently been allocated to wireless communication networks [3] are the reasons why research in the field of millimeter wave communication links has experienced a significant growth in the last ten years. Although important technical challenges, like limited output power and a high noise level, come along with the advantage of the high available bandwidths, experiments with wireless links in this frequency band have been reported. A summary of the state of the art of successful data transmissions, in terms of data rate versus link distance, is presented in Fig. 1.

Data transmissions are classified depending on the used technology. The first category is based on photonic generation of high speed $\mathrm{THz}$ signals. The progress in fiber optics has positively influenced the development of wireless links using photonics-based transmitters [2]. At very high carrier 
TABLE I: State of the art wireless links above $200 \mathrm{GHz}$ using different technologies.

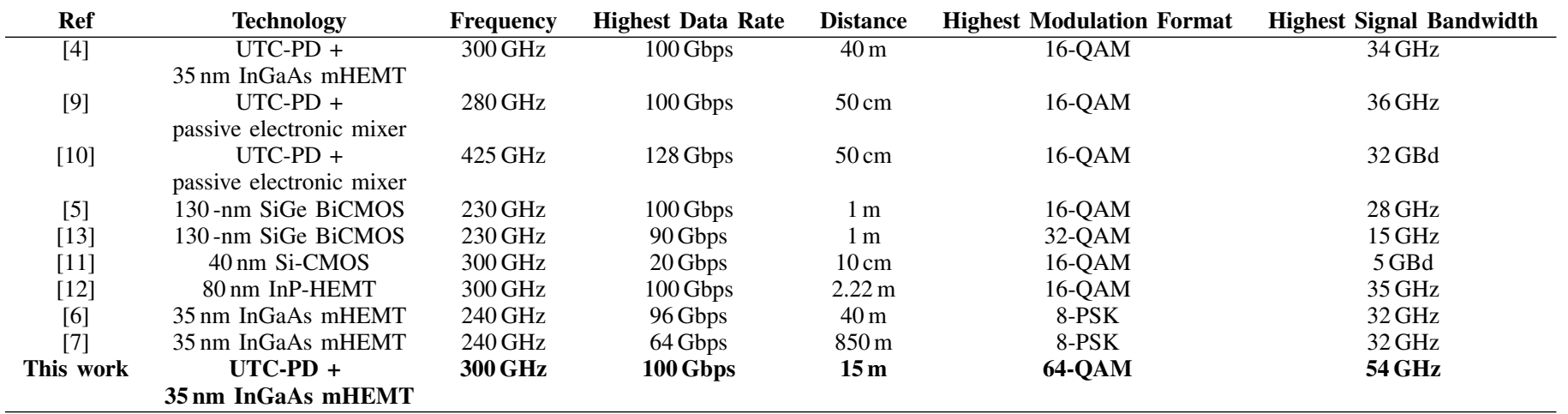

frequencies of 300 and $425 \mathrm{GHz}$, not only very high data rates of up to $128 \mathrm{Gbps}$ can be achieved [9], [10], but also a high distance, up to 40 meters for a transmission of $100 \mathrm{Gbps}$ [4].

There has also been notable progress in the millimeterwave links using the electronic approach, where both the transmitter and the receiver are generated electronically. A recent publication [5] reports on a transceiver using a SiGe hetero-junction bipolar transistor (HBT), which has been successfully used in a data transmission and has reached a data rate of $100 \mathrm{Gbps}$, at a center frequency of $230 \mathrm{GHz}$ and a transmitting distance of $1 \mathrm{~m}$. This data rate is the highest reported in a siliconbased technology. [11] presents a $40 \mathrm{~nm}$ CMOS transceiver which achieves data rates around $20 \mathrm{Gbps}$ over small distances, $10 \mathrm{~cm}$.

Other technologies capable of realizing transceivers that operate above $200 \mathrm{GHz}$ are the III-V compound semiconductor technologies: InP and InGaAs. [12] reports on a $100 \mathrm{Gbps}$ $2.22 \mathrm{~m}$ data transmission using a $80 \mathrm{~nm}$ InP HEMT transceiver. An impressive distance with a corresponding high data rate has been reported in [7]. A transmission over $850 \mathrm{~m}$ was successfully conducted using Quadrature Phase Shift Keying (QPSK) modulation and a symbol rate of $32 \mathrm{GBd}$, which leads to a data rate of $64 \mathrm{Gbps}$. In [6] a transmission over $40 \mathrm{~m}$ with a data rate of $96 \mathrm{Gbps}$ is reported.

The work presented here achieves the highest data rate considering the center frequency and the transmission range. This shows the advantages arising by the combination of photonic transmitter and active electronic receiver technologies: on the one hand the intrinsic high available bandwidth of the photonic source and the compatibility to the existing fiberoptic networks and on the other hand the low noise and the on-chip quadrature channel capabilities of active electronics.

Table I gives a summary of the most important parameters for the state of the art wireless data transmissions realized using different technologies. Next to data rate, distance and center frequency of operation, the used modulation format and the signal bandwidth are important parameters to asses the performance of a wireless link and to evaluate its potential for future usage in communication networks. Applications that could benefit from $\mathrm{THz}$ communication will either use multicarrier configuration, where each channel has a limited bandwidth and therefore a higher modulation format is required, or they could take advantage of the immense bandwidth avail- able in the $300 \mathrm{GHz}$ band and use modulation formats with lower spectral efficiency, reducing the linearity requirements of the transceiver. From the state-of-the-art transmission links presented in Fig. 1, none except this work reports on the usage of modulation formats with a spectral density higher than five. [13] shows a 32 -Quadrature Amplitude Modulation (QAM) transmission at $230 \mathrm{GHz}$, which achieves a data rate of $90 \mathrm{Gbps}$, using a signal bandwidth of $15 \mathrm{GHz}$. If a publication did not mention the signal bandwidth the symbol rate has been used in the comparison in Table I. While most works use signal bandwidths of around $35 \mathrm{GHz}$, the transmission presented in this work achieves the highest reported bandwidth of $54 \mathrm{GHz}$.

\section{Key TEChNOLOGIES}

\section{A. Photonic Transmitter}

The photonics-based transmitter is composed of an unitraveling-carrier photodiode (UTC-PD). This device makes use of a semitransparent top contact for front-side illumination of the photodiode with a $1.55 \mu \mathrm{m}$ wavelength and is already described in [14]. The DC photo response of the UTC-PD with $3 \mu \mathrm{m}$ diameter is $0.1 \mathrm{~A} / \mathrm{W}$. The photodiode is directly connected using a Ground-Signal-Ground (GSG) coplanar access with $50 \mu \mathrm{m}$ signal/ground separation. The output of the probe is waveguide-coupled. A waveguide antenna is used to out-couple the signal into the free-space and a $100 \mathrm{~nm}$ Teflon lens is used to collimate the beam. The $300 \mathrm{GHz}$ carrier is generated by photomixing of two laser lines, where the quality of the laser lines directly impacts the quality of the $\mathrm{THz}$ carrier. The optical lines are at $193.4 \mathrm{THz}$ and $193.1 \mathrm{THz}$, hence a wavelength separation of $2.4 \mathrm{~nm}$ which results in the $300 \mathrm{GHz}$ carrier. The first laser is amplitude/phase-modulated by an I/Q Mach-Zehnder modulator using Lithium Niobate technology. This enable modulation-formats up to 64-QAM and a large signal bandwidth up to the $3 \mathrm{~dB}$ bandwidth of the modulator, which is $25 \mathrm{GHz}$. After the combination of the modulated optical signal, a second optical tone is added, to feed the input of an erbium-doped fiber amplifier (EDFA). The amplified optical signal is then injected in the photomixer.

Initial characterization of the photonics transmitter is done using continuous wave signals in the optical domain. The frequency response as well as the linearity are determined. Fig. 2 shows the output power of the photodiode in the 


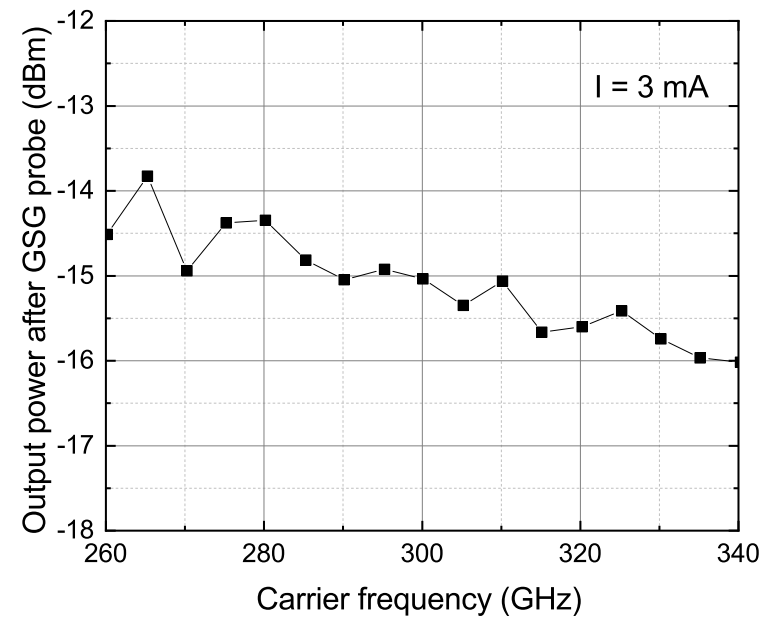

Fig. 2: Frequency evolution of generated power at UTC-PD output, connected to the GSG probe. The power level is measured at waveguide output, for $3 \mathrm{~mA}$ photo current inside the UTC-PD. The photodiode bias is $-1 \mathrm{~V}$.

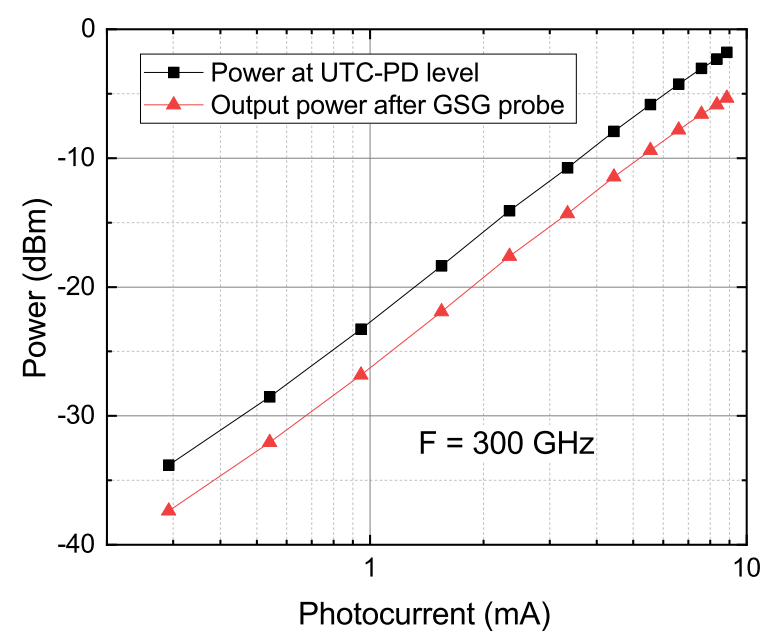

Fig. 3: Linearity of generated power at UTC-PD output for a bias of $-1 \mathrm{~V}$. Red curve: GSG probe output, black: power at UTC-PD output (wafer level). A linear behavior of the UTC-PD is obtained up to approximately $8 \mathrm{~mA}$. In the data communication experiments, a $4 \mathrm{~dB}$ margin (back-off) is taken for linear operation of the UTC. This corresponds to a photocurrent smaller than $5 \mathrm{~mA}$ as in the photomixing process, the generated power is proportional to the square of the photocurrent inside the photodiode.

$300 \mathrm{GHz}$ range with a current level of $3 \mathrm{~mA}$. As can be seen, a broadband behavior is obtained, with a roll-off due to the UTC-PD and probe effect. A 3-dB RF bandwidth of over $80 \mathrm{GHz}$ centered around $300 \mathrm{GHz}$ was obtained, compatible with very high baud-rate modulation.

Fig. 3 presents the linear behavior of the photonics transmitter at a carrier frequency of $300 \mathrm{GHz}$. The red curve shows the measured power at the waveguide (WR3.4) output after GSG probe, which is the actual power radiated in free space using a horn antenna. The black curve shows the UTC-PD power taking into account the probe losses.

\section{B. Electronic Receiver}

The electronic receiver is based on a milimeterwave monolithic integrated circuit (MMIC) fabricated using a $35 \mathrm{~nm}$ metamorphic high electron mobility transistor (mHEMT) by Fraunhofer IAF. This technology is best suited for fabricating $\mathrm{THz}$ receivers due to its excellent low noise properties and very high transit frequency $f_{\mathrm{t}}$ above $500 \mathrm{GHz}$ and maximum frequency of oscillation $f_{\max }$ above $1 \mathrm{THz}$ [15].

The receiver integrates a frequency multiplier by three, a buffer amplifier, a fundamental passive IQ mixer and a threestage low-noise amplifier. Fig. 4 shows the schematic of the receiver. The single stages of the circuit have been described in detail in [16] and [8]. The integrated receiver is assembled in a waveguide split-block module. The average conversion gain of the receiver lies at $6.5 \mathrm{~dB}$ from 270 to $325 \mathrm{GHz}$. The measured room temperature noise figure lies at an average of $8.6 \mathrm{~dB}$ [16]. With the help of a sensitivity measurement described in detail in [8] the optimal input power of the receiver is determined at $-41 \mathrm{dBm}$. Therefore, the power that reaches the receiver should lie in this range and the transmitting distance and gain of the antennas have to be chosen accordingly.

\section{Digital Signal Processing}

The transmitted signal is generated by an Arbitrary Waveform Generator (AWG) with 8 bit resolution, $25 \mathrm{GHz}$ analog bandwidth per channel and a maximum sampling rate of $65 \mathrm{GSa} / \mathrm{s}$. The complex in-phase and quadrature (I/Q) data signal is numerically generated as follows: a pseudo random binary sequences (PRBS) with a length of $2^{15}-1$ is converted to an integer sequence and mapped over a QAM constellation. The resulting complex sequence is up-sampled to match the AWG sampling rate range and filtered using a raised-cosine digital filter with different roll-off factors $\alpha$. In comparison to rectangular pulses, which have a theoretically infinitely broadband spectrum and will influence other frequency bands, raised cosine pulse shaping offers a low adjacent channel interference. Considering a double-sideband transmission the bandwidth (BW) of the occupied RF signal can be defined using

$$
\mathrm{BW}=\text { Symbolrate } \cdot(1+\alpha)
$$

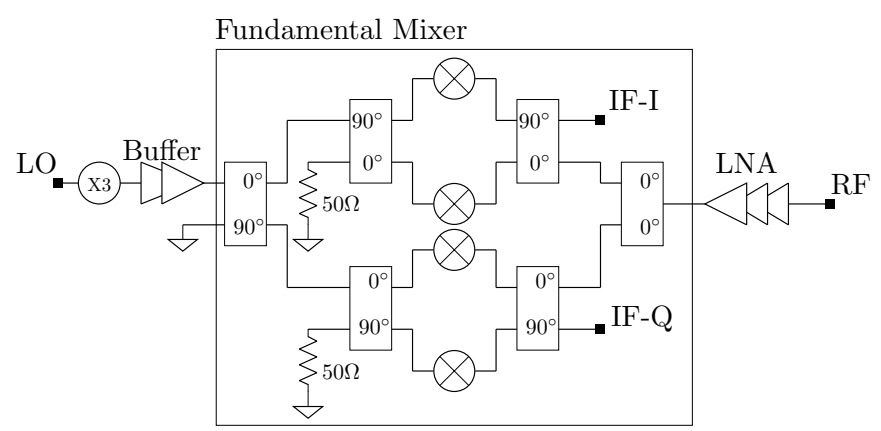

Fig. 4: Schematic of the active electronic receiver integrating a frequency multiplier by three, a buffer amplifier, a fundamental passive IQ mixer and a three stage LNA. 


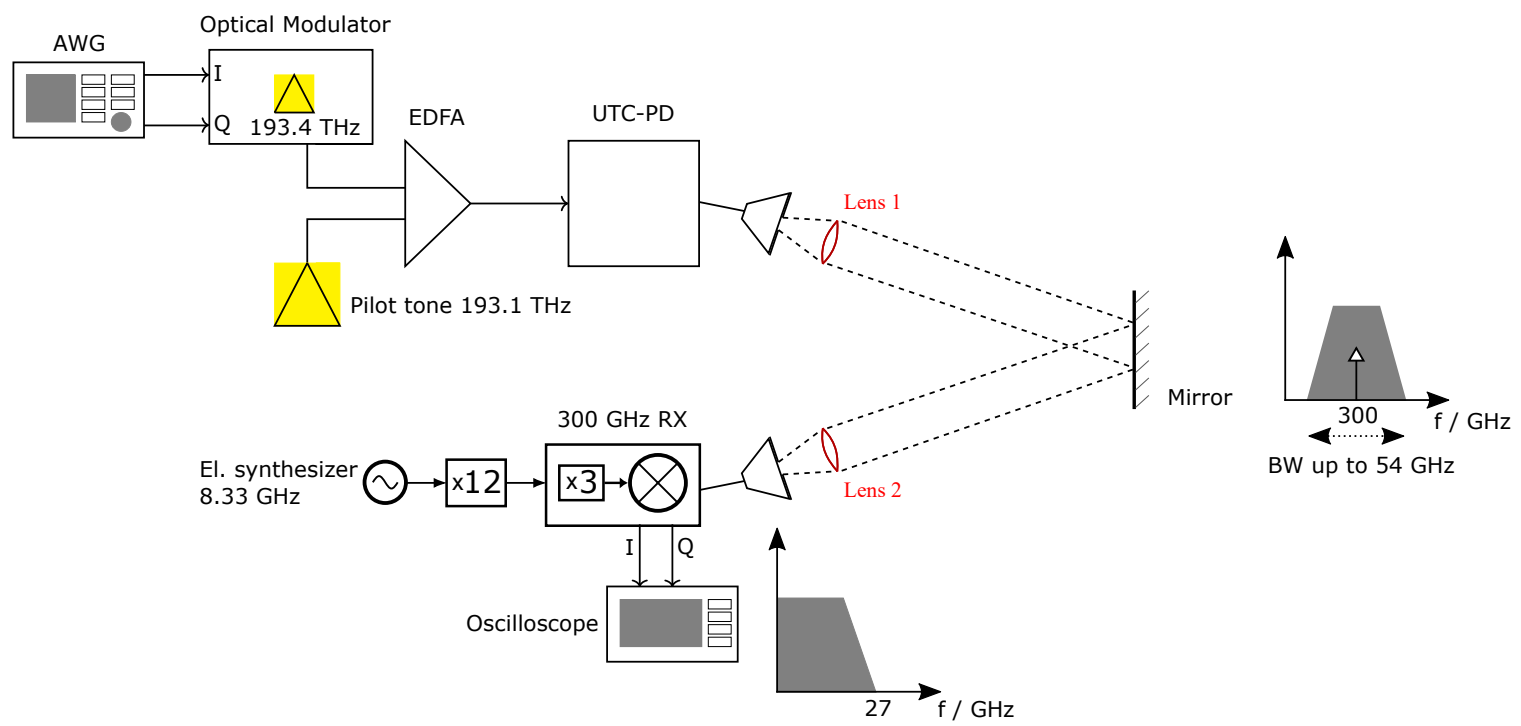

Fig. 5: Schematic of the wireless link setup. A mirror is placed between the UTC-PD transmitter and receiver, doubling the transmitting distance. A brief spectrum of the RF signal as well as of the incoming IF signal is presented.

Therefore, if we consider a data signal with a symbol rate of $32 \mathrm{GBd}$ and a filter with an $\alpha$ of 0.35 , we achieve an RF signal bandwidth of $54 \mathrm{GHz}$.

At the receiver side a real time oscilloscope with $80 \mathrm{GSa} / \mathrm{s}$, an analog bandwidth of $32 \mathrm{GHz}$ and 8 bit vertical resolution captures the I- and Q-signals from the receiver. Since the transmitter and the receiver do not share the same local oscillator signal source and no hardware carrier recovery is used, the setup is incoherent. A vector signal analyzer software analyzes the incoming signal and performs the carrier recovery and the frequency equalization to compensate for the frequency and phase drift along the link. The digital equalization tool of the software has been applied to all the results shown in this paper.

\section{SETUP OF THE WIRELESS LiNKS}

Fig. 5 shows the schematic setup of the data transmission. The signal is radiated into free space and transmitted over a distance of $15 \mathrm{~m}$. For this purpose a mirror is placed at half the distance. This provides a convenient placement of the receiver next to the transmitter. A system of two standard horn antennas and collimating $100 \mathrm{~nm}$ Teflon lenses are used to align the quasi-optical link between TX and RX. The receiving system is composed of two modules: the $300 \mathrm{GHz}$ receiver module and the multiplier by twelve module, which provides the $100 \mathrm{GHz}$ LO signal necessary for the receiver. An electronic synthesizer provides the $8.33 \mathrm{GHz}$ LO input signal. Fig. 6 shows photographs of the UTC-PD photomixer and of the $300 \mathrm{GHz}$ electronic receiver.

In order to exploit the maximum distance of $15 \mathrm{~m}$ available in the laboratory, which results in $105.5 \mathrm{~dB}$ of FSPL and considering the optimal input receiving power $\mathrm{P}_{\mathrm{rf}, \text { in }}$ of around $-41 \mathrm{dBm}$ the sum of transmitted power $\mathrm{P}_{\text {out }, \mathrm{TX}}$ and antenna gain $\mathrm{G}_{\mathrm{A}}$, lies as calculated in (2) at around $65 \mathrm{dBm}$.

$$
\begin{aligned}
\mathrm{P}_{\text {out }, \mathrm{TX}}+2 \cdot \mathrm{G}_{\mathrm{A}} & =\mathrm{P}_{\mathrm{rf}, \text { in }}+\mathrm{FSPL} \\
& =-41 \mathrm{dBm}+105.5 \mathrm{~dB} \\
& =64.5 \mathrm{dBm}
\end{aligned}
$$

\section{WIRELESS DATA TRANSMISSION}

To characterize the $300 \mathrm{GHz}$ analog transmit-receive wireless system in terms of bandwidth and gain imbalance, the measurement of the complex transfer function of the system was conducted. For this purpose, a vector network analyzer (VNA) measures the S-Parameters of the transmit-receive system. The setup of the measurement is similar to the one shown in Fig 5, with the only difference that the AWG and the oscilloscope are replaced by the VNA. The signal originating from the first port of the VNA, connected to the I-channel of the optical modulator is up-converted by the UTC-PD and transmitted over the air to the electronic receiver, downconverted and received on the I-channel, which is connected to the second port of the VNA. The Q-channels are terminated with $50 \Omega$ loads. The same measurement is conducted by connecting the ports of the VNA to the Q-channels of the modulator and of the receiver. A detailed explanation of this measurement is presented in [17].
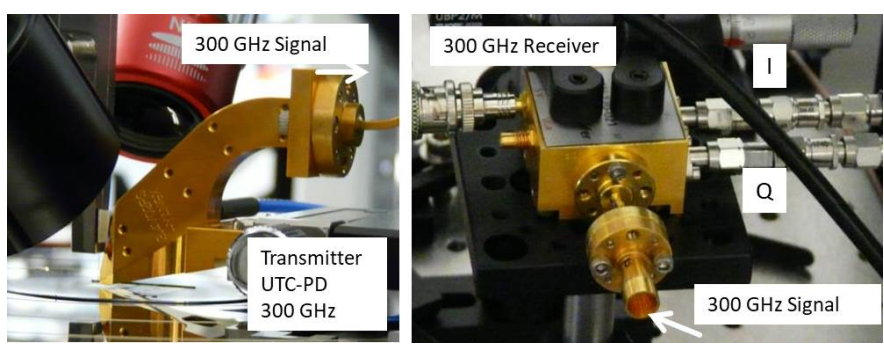

Fig. 6: Photographs of the UTC-PD transmitter and electronic receiver with horn antenna and I/Q connections. 


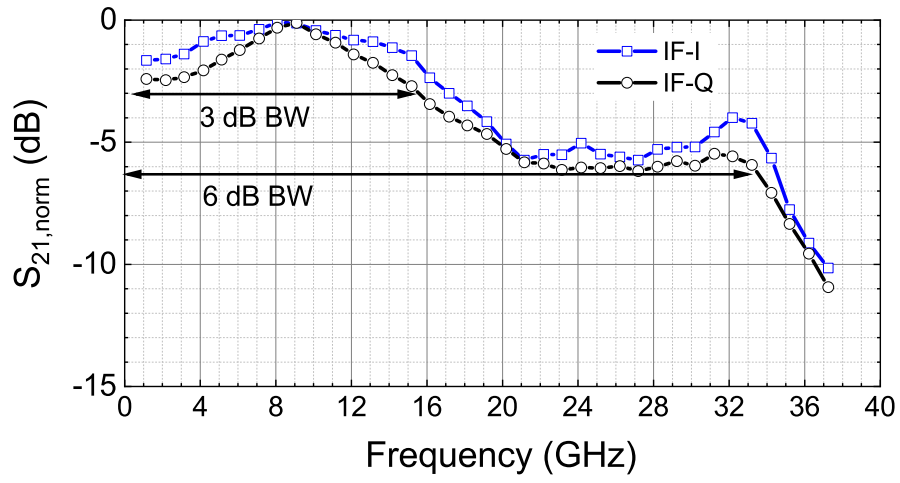

Fig. 7: Measurement results of the transfer function characteristics, when the transmission is done from the I-channel to the I-channel or from Q-channel to Q-channel.

Since the setup is incoherent, a phase shifter is placed after the electronic synthesizer and adjusted in a way that the maximum power is in the measured channel and therefore compensating for the total phase delay introduced in the upand down-converter.

In comparison to on-chip measurements, this measurement shows the overall frequency response of the system and the effective bandwidth limitation. The characteristics shown in Fig. 7 for the I-I and Q-Q transmission represent the linear transfer gain, when both the transmitter and the receiver are operated in their linear region. The transmission parameter $\mathrm{S}_{21}$ is normalized to its highest value for a better representation of the bandwidth limitation. While the $3 \mathrm{~dB}$ IF bandwidth lies at $16 \mathrm{GHz}$, the $6 \mathrm{~dB}$ bandwidth exceeds $30 \mathrm{GHz}$, which results in an RF bandwidth of over $60 \mathrm{GHz}$.

\section{A. 100 Gbps Wireless Data Transmission}

After the measurement of the analog transfer function of the transmit-receive system, data transmission experiments have been carried out. Signals with different modulation formats, symbol rates and digital filtering were transmitted over the distance of $15 \mathrm{~m}$ and the quality of the transmission has been analyzed.

Fig. 8 shows the constellation diagrams and the power spectra of 16-QAM modulated signals with increasing symbol rates. The black dots in the constellation diagrams represent the results of the demodulation of a reference signal. Prior to the $300 \mathrm{GHz}$ wireless data experiment, signals with different modulation formats and symbol rates were measured by connecting the signal coming from the optical modulator directly to the oscilloscope. Therefore, the black dots and the resulting EVM are the best possible result and represent the reference signal.

Fig. 8a shows the results for a symbol rate of $4 \mathrm{GBd}$, resulting in $16 \mathrm{Gbps}$. For this low data rate the constellation diagram is very clear and the EVM deterioration in comparison to the reference signal is below $5 \%$. An increase in bandwidth and symbol rate leads to a decrease in the frequency response of the overall system demonstrated by the frequency dependency of the $S_{21, \text { norm }}$ transmission parameter presented in Fig. 7. For a symbol rate of $12.5 \mathrm{GBd}$ shown in Fig. $8 \mathrm{~b}$, the EVM deterioration lies slightly above $5 \%$. The bandwidth occupied by the $12.5 \mathrm{GBd} 16-\mathrm{QAM}$ modulated signal is within the $3 \mathrm{~dB}$ bandwidth of the system presented in Fig. 7.

Although the absolute EVM value of the measured $20 \mathrm{GBd}$ signal, Fig. 8c, is above $12 \%$ the EVM deterioration is similar to the previous cases, around $5 \%$. The occupied IF-bandwidth of $15 \mathrm{GHz}$ matches the $3 \mathrm{~dB}$ bandwidth of the $300 \mathrm{GHz}$ wireless system. For the signal that achieved the highest data rate, Fig. $8 \mathrm{~d}$, the EVM deterioration is above $10 \%$. This nonlinear increase points to a surpassing of the $3 \mathrm{~dB}$ bandwidth and to increasing effects of impairments like LO isolation. For an Additive White Gaussian Noise (AWGN) channel, the SNR limit for 16-QAM modulation and a bit error rate (BER) smaller than $4 \cdot 10^{-3}$, which can be reduced to $10^{-15}$ using forward error correction codes [18] is $13.4 \mathrm{~dB}$. The $25 \mathrm{GBd}$ signal reaches an SNR of $12.4 \mathrm{~dB}$ and is therefore below this limit and could not be successfully demodulated. All the other signals, $20 \mathrm{GBd}, 12.5 \mathrm{GBd}$ and $4 \mathrm{GBd}$, have an SNR of $15.5 \mathrm{~dB}, 17.2 \mathrm{~dB}$ and $20 \mathrm{~dB}$ and represent successful transmissions.

An impairment that negatively influences the transmission of the $25 \mathrm{~dB}$ signal is the LO to IF isolation, which can be graphically observed in the spectra in Fig. 8 at $16.66 \mathrm{GHz}$. The spectral lines at $8.33 \mathrm{GHz}, 16.66 \mathrm{GHz}$ and their negative correspondents are parasitic spurious tones of undesired harmonics. The mechanical interface between the multiplier module and the $300 \mathrm{GHz}$ receiver is a WR-10 waveguide. This means that only harmonics above the lower cutoff frequency of the WR10 waveguide with an order higher than $8(\mathrm{X} 8=8 \cdot 8.33 \mathrm{GHz}=$ $66.64 \mathrm{GHz}$ ) can propagate. Considering the buffer amplifier's limited bandwidth, which acts as a filter for most undesired harmonics, only a few combinations remain plausible for the cause of the leakage. Fig. 9 shows the spectral density of the leaked signal, when the RF signal is turned off. Therefore, only the leakage of the LO in the receiver can be observed. The rest of the measurement setup remains unchanged, the demodulation occurs using both I and Q outputs, hence the possibility to plot also the negative part of the spectrum. The mixing of the 39th harmonic (X39), the leakage of the 13th harmonic coming from the multiplier module multiplied by three in the receiver, and the 40th harmonic (X40), an undesired 4th harmonic of the 10th harmonic coming from the multiplier module cause a first spurious tone at $8.33 \mathrm{GHz}$. The highest leakage, at $16.66 \mathrm{GHz}$, is caused by the mixing of the 36th harmonic (X36) at $300 \mathrm{GHz}$ and the 34th harmonic (X34). $\mathrm{X} 34$ is an undesired 2nd harmonic of the 17th harmonic (X17) coming from the multiplier by twelve module. A less likely possibility is the mixing of the 40th harmonic (X40) with the 42nd harmonic (X42), which at $349.86 \mathrm{GHz}$ is strongly filtered out by the buffer amplifier and can most probably not cause such a high leakage.

The asymmetry between the positive and the negative part of the spectrum has its origin in the particularity of the fundamental mixer, shown in Fig. 4. The IF signal is measured at the isolated port of the last $90^{\circ}$ coupler and not, as usual, directly at the IF mixer output. In addition, only one of the mixer paths in the I- and one in the Q-channel is used. Despite this, the non-linear mixing products of both paths are added in 

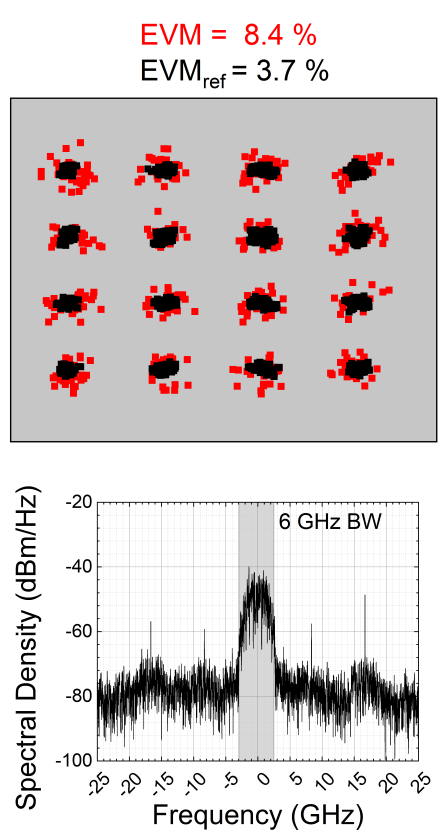

(a)
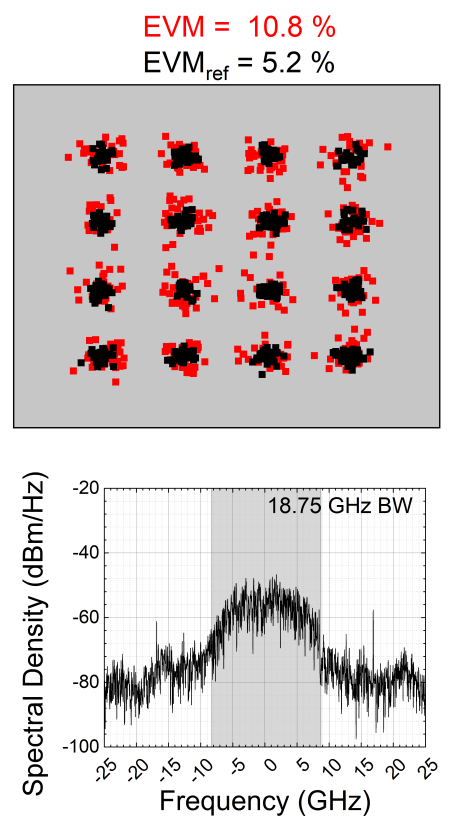

(b)
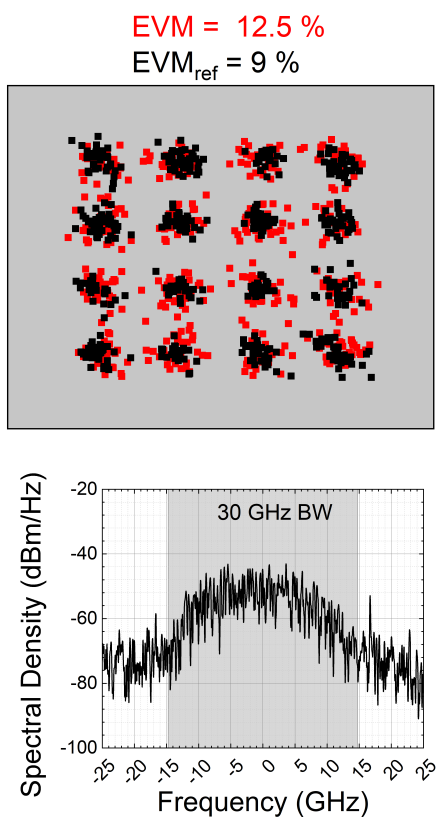

(c)
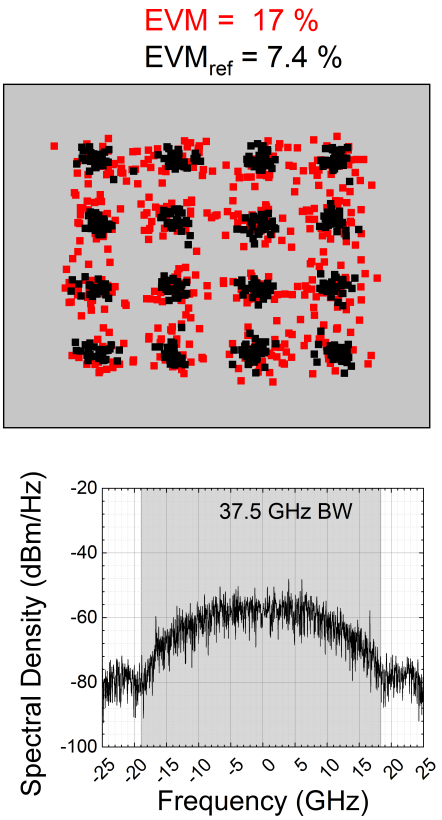

(d)

Fig. 8: Constellation diagrams and the corresponding spectral densities of 16-QAM modulated signals with increasing symbol and data rates. (a) $4 \mathrm{GBd}, 16 \mathrm{Gbps}$. (b) $12.5 \mathrm{GBd}, 50 \mathrm{Gbps}$. (c) $20 \mathrm{GBd}, 80 \mathrm{Gbps}$. (d) $25 \mathrm{GBd}, 100 \mathrm{Gbps}$. The black dots represent the results of a reference signal measured by connecting the signal coming from the optical modulator directly to the oscilloscope and the red dots represent the results of the actual signal transmitted over $15 \mathrm{~m}$ using the setup in Fig. 5.

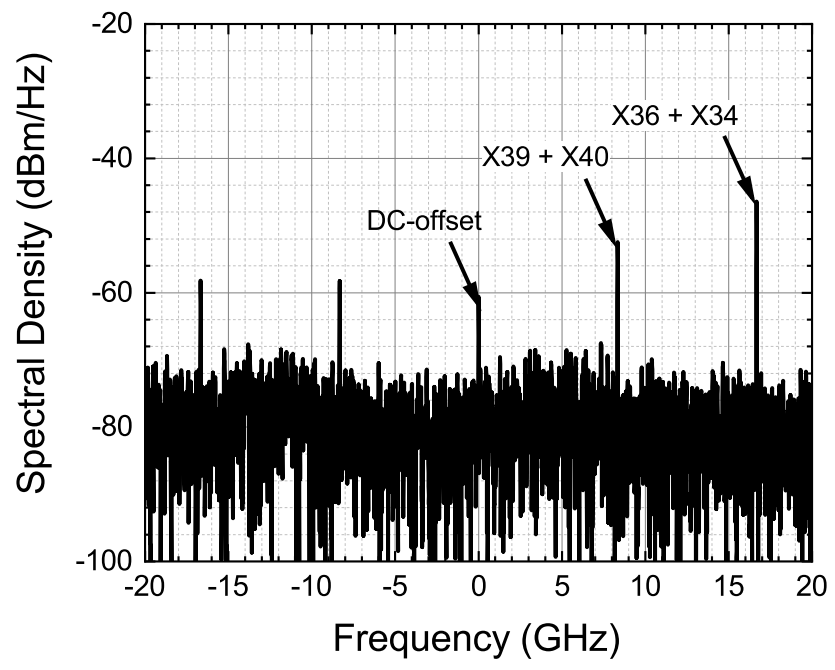

Fig. 9: Power spectrum measured without an RF signal, showing the LO leakage in the receiver.

the coupler, thus producing a different phase shift. The leakage measured on I and Q are demodulated in the VSA software, which leads to the asymmetry in the spectrum.

While for the first three 16-QAM transmissions in Fig. 8a to Fig. 8c the LO leakage is outside the bandwidth of the signal, depicted by the gray area, in the last case, Fig. $8 \mathrm{~d}$ the peak is inside the desired signal, which leads to an EVM deterioration. To avoid this and to improve the quality of the transmission, a narrower digital filter is used, with a roll-off factor of 0.35 instead of 0.5 , which reduces the bandwidth by $4.25 \mathrm{GHz}$.

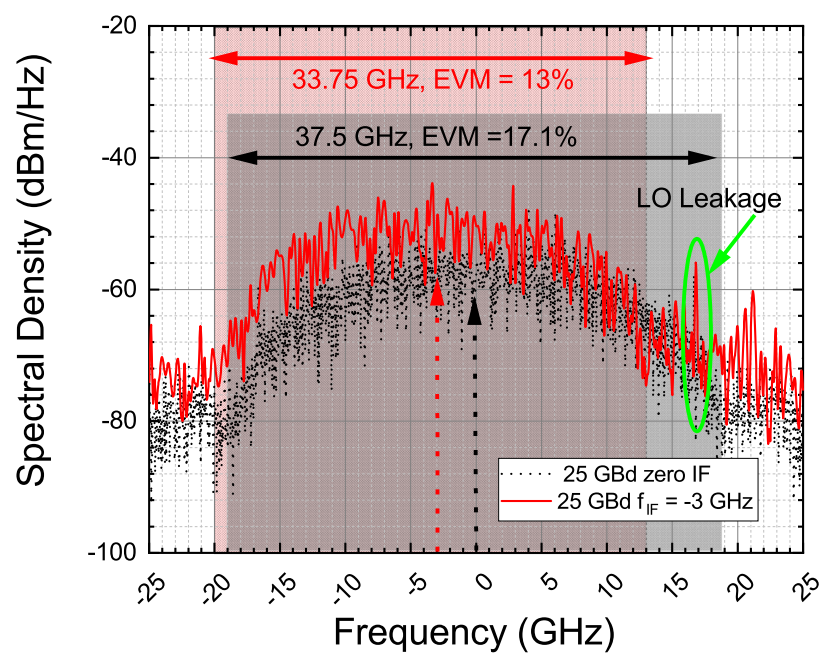

Fig. 10: Comparison between power spectrum of a $25 \mathrm{GBd}$ 16-QAM modulated signal with different bandwidths and center frequencies.

Additionally, the IF signal is modulated using an intermediate frequency generated in the AWG, so that the signal is centered around $-3 \mathrm{GHz}$.

Fig. 10 shows the comparison of the power spectra between a $25 \mathrm{GBd}$ 16-QAM modulated signal with a bandwidth of $37.5 \mathrm{GHz}$ represented in the dotted black curve, and one with a bandwidth of $33.75 \mathrm{GHz}$ centered around $-3 \mathrm{GHz}$ represented in the solid red curve. The signal level of the more narrowband signal is higher than the one of the more broadband 
16-QAM, 4 GBd, 16 Gbps

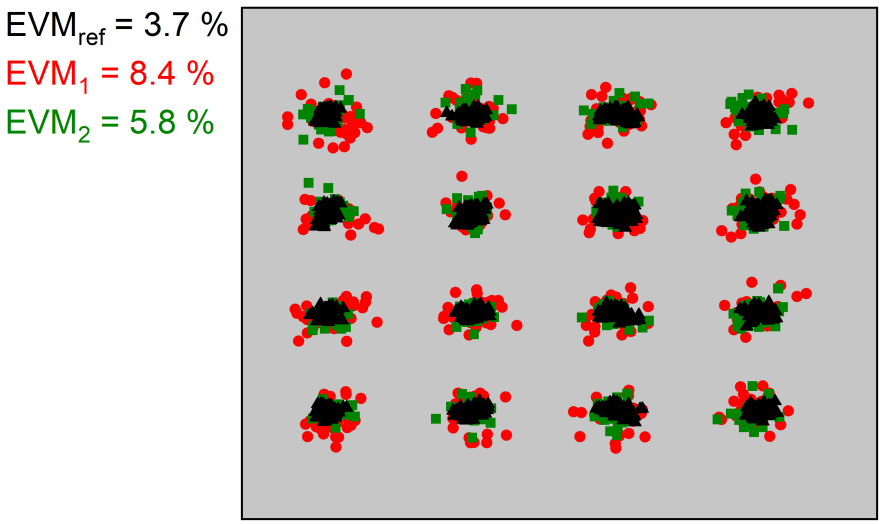

Fig. 11: Graphic representation of the EVM improvement due to LO leakage reduction and shifting of the transmission center frequency. The black dots represent the reference signal, the red dots are the original transmitted 16QAM signal and green the improved transmission.

signal. In the case of the narrow-band signal the LO leakage is outside the signal bandwidth. All these lead to an EVM improvement of more than $4 \%$. The final EVM absolute value is therefore $13 \%$, which are above the 16-QAM limit for BER smaller than $4 \cdot 10^{-3}$. Thus, $100 \mathrm{Gbps}$ wireless transmission has been successfully proven. Comparing the bandwidth achieved for this very high data rate with the frequency response of the overall system, the conclusion can be derived that for 16-QAM modulation the 3-dB bandwidth is the limit for a successful transmission.

A 16-QAM 4 GBd signal is transmitted using the changes described above: a signal centered around a given non-zero IF frequency and a filter with a roll-off factor of 0.35. Fig. 11 shows the constellation diagram of this new transmission in green and the comparison to the reference signal in black and the zero IF signal in red. Compared to the signal in Fig. 8a the results of this new transmission show an improvement in EVM of $31 \%$, leading to an absolute EVM value of $5.8 \%$ and an improvement in SNR of $14.4 \%$, leading to an absolute SNR value of $22.08 \mathrm{~dB}$.

\section{B. Transmission of Complex Modulation Formats}

In order to show the potential of the presented wireless link for future high data rate applications, signals using complex modulation formats were transmitted over the air. Data-hungry applications like kiosk-downloading, data-centers and smart offices use either a very broadband signal modulated with simple modulation formats or they aggregate multiple channels, each with a narrow bandwidth, but with complex modulation formats.

Fig. 12 and Fig. 13 show the constellation diagrams and the corresponding spectral densities of successfully transmitted signals with higher order modulation formats which achieved the highest data rate. For 64-QAM, a signal with a symbol rate of $5 \mathrm{GBd}$, filtered using a root-raised cosine filter with a roll-off factor of 0.35 and centered around a non-zero

\section{4-QAM, 5 GBd, 30 Gbps}
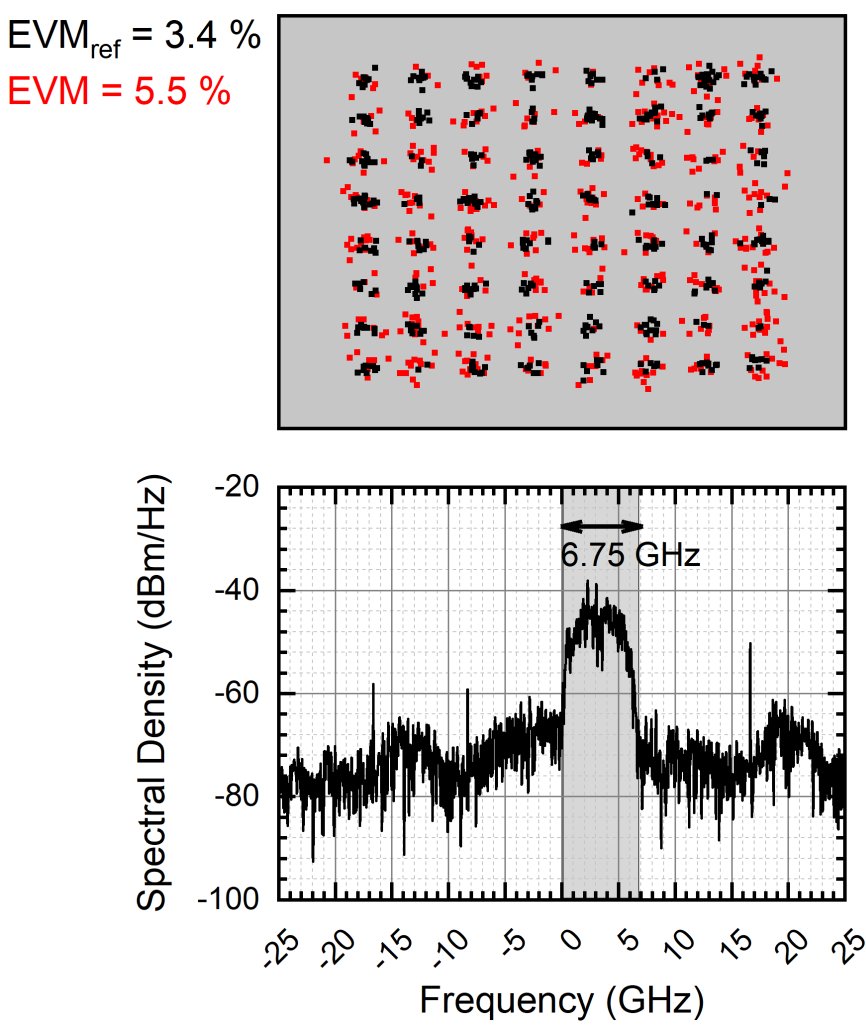

Fig. 12: Constellation diagram and the corresponding spectral density of a 64-QAM modulated signal with a symbol rate of $5 \mathrm{GBd}$ and bandwidth of $6.75 \mathrm{GHz}$ achieving a data rate of 30 Gbps.

intermediate frequency achieves an absolute SNR value of $21.4 \mathrm{~dB}$. For an AWGN channel, this value is above the limit for 64-QAM and BER smaller than $4 \cdot 10^{-3}$, which lies at $17.8 \mathrm{~dB}$. Hence, the transmission of $64-\mathrm{QAM}, 5 \mathrm{GBd}$ is successfully demodulated. Compared to the reference signal, the EVM value deteriorates by slightly more than $2 \%$, which is comparable to the deterioration for 16-QAM modulation at similar symbol rates shown in Fig. 8a. Due to the high linearity requirements of this modulation format, this is the highest data rate achieved with 64-QAM. Looking at the measurement of the transfer function in Fig. 7 we observe that the frequency response is flat until $3 \mathrm{GHz}$, which coincides with the IF bandwidth limit for 64-QAM. Also, at $4 \mathrm{GHz}$, there is a peak in the IQ-imbalance curve. A lower IQ-amplitude imbalance, a flatter frequency response of the system and a higher isolation of the LO leakage can help increase the transmitting bandwidth and thus, improve the highest possible data rate for complex modulation formats.

Another important aspect is that the distance between transmitter and receiver is kept constant during all the transmission experiments. The optimal input receiving power was determined and set for 16-QAM. To optimize the 64-QAM transmission a dynamic change in distance and transmitting output power is needed. Due to constraints of the mechanical indoor setup the transmitting distance could not be changed. 


\section{2-QAM, 8 GBd, 40 Gbps}
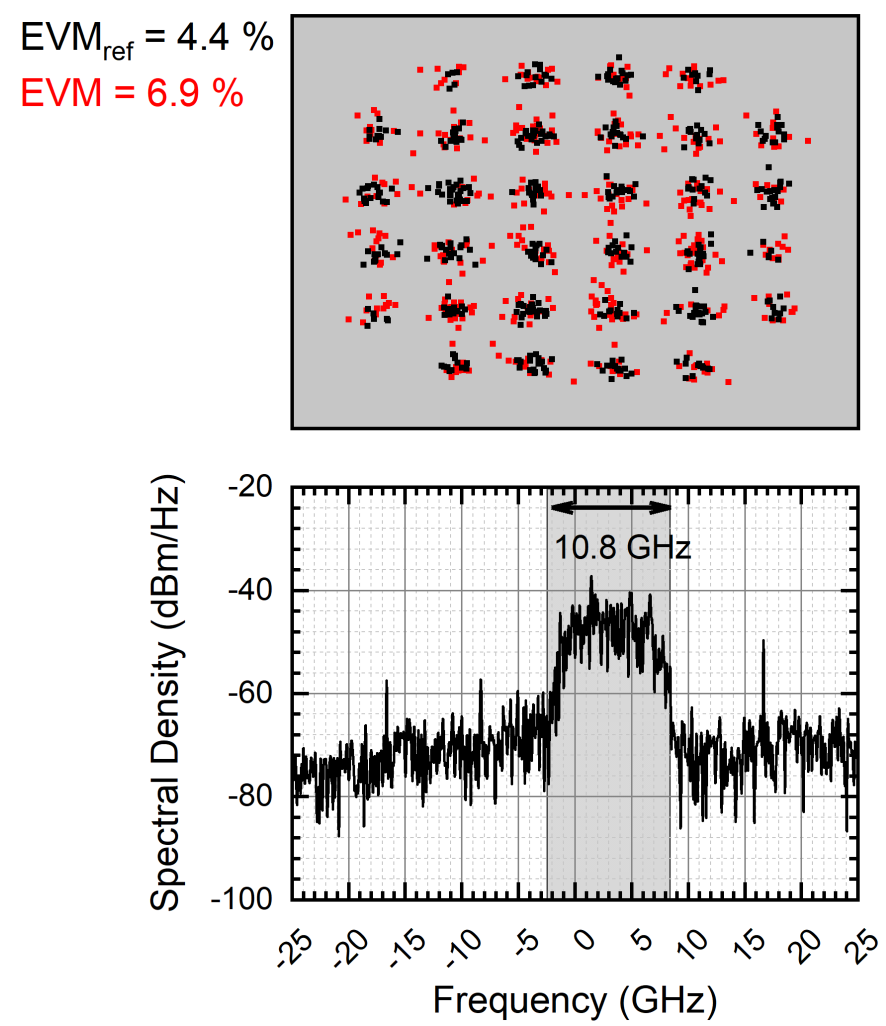

Fig. 13: Constellation diagram and the corresponding spectral density of a 32-QAM modulated signal with a symbol rate of $8 \mathrm{GBd}$ and bandwidth of $10.8 \mathrm{GHz}$ achieving a data rate of 40 Gbps.

For 32-QAM modulation format a bandwidth of up to $10.8 \mathrm{GHz}$ is successfully transmitted. The results of this transmission are presented in Fig. 13. An EVM value of around $7 \%$ and an EVM deterioration of $2.5 \%$ are achieved. The fact that a higher bandwidth than in the case of 64-QAM was reached and the transfer function characteristic points out that for 32QAM a 1 to $2 \mathrm{~dB}$ deviation from a perfectly flat frequency response and an IQ imbalance of up to $1 \mathrm{~dB}$ are acceptable.

\section{Transmission of Signals with High Bandwidth}

Using simple modulation formats, like binary phase shift keying (BPSK) and quadrature phase shift keying (QPSK), the limits of the wireless system in terms of bandwidth can be tested. This approach, transmitting very broadband signals in one channel, is a viable alternative to systems using multi-channels and complex modulation formats and has been adopted in most previous works [2] - [13].

The $300 \mathrm{GHz}$ wireless link presented in this work successfully transmitted and received signals with RF bandwidth up to $54 \mathrm{GHz}$ using QPSK modulation format. Fig. 14 shows the dependency between EVM deterioration and signal bandwidth. The EVM deterioration is the difference between the measured EVM for the actual signal and the EVM value of the reference signal.

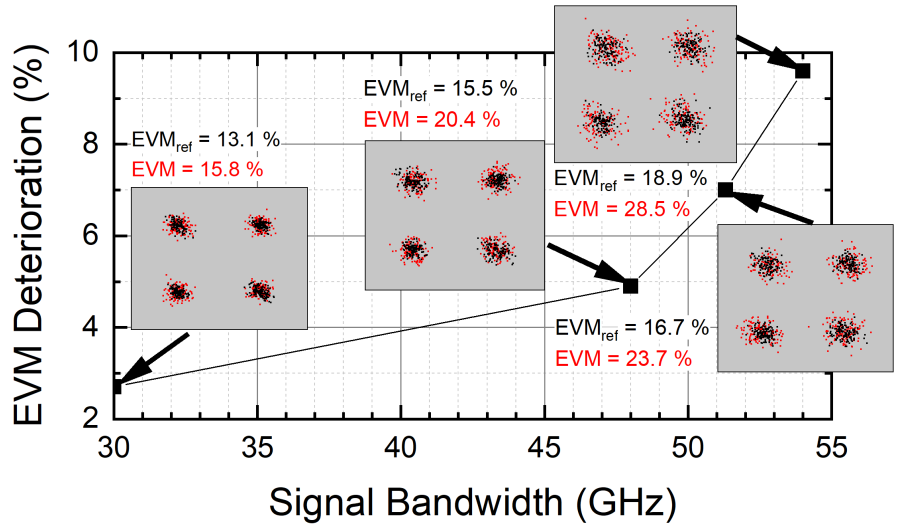

Fig. 14: EVM deterioration and constellation diagrams for QPSK modulated signals with increasing symbol rates and bandwidth.

The $54 \mathrm{GHz}$ RF bandwidth was achieved with a signal with a symbol rate of $40 \mathrm{GBd}$. The absolute value of the SNR is $10.9 \mathrm{~dB}$, which is slightly above the successful demodulation limit for QPSK and AWGN channel, SNR of $9.6 \mathrm{~dB}$. While for a signal with $20 \mathrm{GBd}$ symbol rate and $30 \mathrm{GHz}$ of bandwidth, the EVM value differs just by $3 \%$ from the reference, signals with bandwidths above $45 \mathrm{GHz}$ show a substantial deterioration of almost $10 \%$ at $54 \mathrm{GHz}$. The frequency response of the system measured and presented in Fig. 7 shows that between $24 \mathrm{GHz}$ and $33 \mathrm{GHz}$ IF frequency the $6 \mathrm{~dB}$ bandwidth limit is reached. The successfully transmitted QPSK signal occupies $27 \mathrm{GHz}$ of IF bandwidth. This means that for QPSK modulation format the digital equalization tool can compensate up to a limit of $6 \mathrm{~dB}$ from the frequency response of the system. Other limiting factors are the IQ phase and amplitude imbalances, which can be observed in the constellation diagrams, where the spread of the red points worsens with increasing bandwidth.

\section{CONCLUSiON}

A $15 \mathrm{~m}$ wireless link operating at a center frequency of $300 \mathrm{GHz}$ is presented. The link combines two different technologies, a photonic transmitter and an electronic active receiver, and reaches data rates of up to $100 \mathrm{Gbps}$. Compared to other works, this is the highest data rate achieved for this frequency and range. In addition to a high data rate, the link shows a great potential in usage of a multi-channel approach for future wireless applications. Complex modulation formats like 32-QAM and 64-QAM are successfully transmitted for bandwidths of up to $10 \mathrm{GHz}$. The transmission bandwidth of the system exceeds $54 \mathrm{GHz}$, this being the highest ever reported transmission bandwidth.

Future work involves an improvement in the analog frontends non-idealities, LO leakage and IQ imbalances. This would lead to both a higher data rate and higher transmitting distance, which can open new application possibilities.

\section{ACKNOWLEDGMENT}

This work was funded by the Agence Nationale de la Recherche (ANR) within the TERASONIC project, grant CE- 
24, APPG-17 and the German Deutsche Forschungsgemeinschaft (DFG) under grant number KA3062/12-1. We express our gratitude to the colleagues at Fraunhofer Institute of Applied Solid-State Physics for MMIC manufacturing and for providing the modules. We also thank the IEMN NanoMicrowave RF/MEMS characterization centre and the IEMNIRCICA Telecom platform facilities. This work is supported by the CPER 'Photonics for society' supported by the Hautsde-France regional council, and contributes to the "digital world" Hub 3 of the I-Site Université de Lille Nord de France.

\section{REFERENCES}

[1] T. Nagatsuma and G. Carpintero, "Recent Progress and Future Prospect of Photonics-Enabled Terahertz Communications Research," IEICE Transactions on Electronics, 2015.

[2] G. Ducournau, P. Szriftgiser, A. Beck, D. Bacquet, F. Pavanello, E. Peytavit, M. Zaknoune, T. Akalin, and J. F. Lampin, "Ultrawide-Bandwidth Single-Channel 0.4-THz Wireless Link Combining Broadband QuasiOptic Photomixer and Coherent Detection," IEEE Transactions on Terahertz Science and Technology, vol. 4, no. 3, pp. 328-337, May 2014.

[3] IEEE Standard for High Data Rate Wireless Multi-Media Networks Amendment 2: 100 Gbps Wireless Switched Point-to-Point Physical Layer, IEEE-SA Standards Board Std.

[4] S. Koenig, D. Lopez-Diaz, J. Antes, F. Boes, R. Henneberger, A. Leuther, A. Tessmann, R. Schmogrow, D. Hillerkuss, R. Palmer, T. Zwick, C. Koos, W. Freude, O. Ambacher, J. Leuthold, and I. Kallfass, "Wireless sub-THz Communication System with High Data Rate Enabled by RF Photonics and Active MMIC Technology," in 2014 IEEE Photonics Conference, Oct 2014, pp. 414-415.

[5] P. Rodríguez-Vázquez, J. Grzyb, B. Heinemann, and U. R. Pfeiffer, "A 16-QAM 100-Gbps 1-m Wireless link with an EVM of 17an SiGe technology," IEEE Microwave and Wireless Components Letters, vol. 29, no. 4, pp. 297-299, April 2019.

[6] F. Boes, T. Messinger, J. Antes, D. Meier, A. Tessmann, A. Inam, and I. Kallfass, "Ultra-broadband MMIC-based wireless link at $240 \mathrm{GHz}$ enabled by 64GSpros DAC," in 2014 39th International Conference on Infrared, Millimeter, and Terahertz waves (IRMMW-THz), Sept 2014, pp. 1-2.

[7] I. Kallfass, B. Florian, M. Tobias, A. Jochen, I. Anns, L. Ulrich, T. Axel, and H. Ralf, "64 Gbps transmission over 850 m Fixed Wireless Link at $240 \mathrm{Ghz}$ Carrier Frequency," Journal of Infrared, Millimeter, and Terahertz Waves, vol. 36, no. 2, pp. 221-233, 2015. [Online]. Available: http://dx.doi.org/10.1007/s10762-014-0140-6

[8] I. Kallfass, I. Dan, S. Rey, P. Harati, J. Antes, A. Tessmann, S. Wagner, M. Kuri, R. Weber, H. Massler, A. Leuther, T. Merkle, and T. Kürner "Towards MMIC-based $300 \mathrm{GHz}$ Indoor Wireless Communication Systems," IEICE Transactions on Electronics, vol. E98-C, no. 12, pp. 10811090, Dec 2015.

[9] V. K. Chinni, P. Latzel, M. Zégaoui, C. Coinon, X. Wallart, E. Peytavit, J. F. Lampin, K. Engenhardt, P. Szriftgiser, M. Zaknoune, and G. Ducournau, "Single-channel $100 \mathrm{Gbit} / \mathrm{s}$ transmission using III-V UTC-PDs for future IEEE 802.15.3d wireless links in the $300 \mathrm{GHz}$ band," Electronics Letters, vol. 54, no. 10, pp. 638-640, 2018.

[10] S. Jia, X. Pang, O. Ozolins, X. Yu, H. Hu, J. Yu, P. Guan, F. D. Ros, S. Popov, G. Jacobsen, M. Galili, T. Morioka, D. Zibar, and L. K. Oxenløwe, "0.4 THz Photonic-Wireless Link with 106 Gbps Single Channel Bitrate," Journal of Lightwave Technology, vol. 36, no. 2, pp. 610-616, Jan 2018.

[11] S. Hara, K. Takano, K. Katayama, R. Dong, S. Lee, I. Watanabe, N. Sekine, A. Kasamatsu, T. Yoshida, S. Amakawa, and M. Fujishima, "300-GHz CMOS Transceiver for Terahertz Wireless Communication," in 2018 Asia-Pacific Microwave Conference (APMC), Nov 2018, pp. 429-431.

[12] H. Hamada, T. Fujimura, I. Abdo, K. Okada, H. Song, H. Sugiyama, H. Matsuzaki, and H. Nosaka, "300-GHz 100 Gbps InP-HEMT Wireless Transceiver Using a 300-GHz Fundamental Mixer," in 2018 IEEE/MTTS International Microwave Symposium - IMS, June 2018, pp. 1480-1483.

[13] P. Rodríguez-Vázquez, J. Grzyb, B. Heinemann, and U. R. Pfeiffer, "Performance evaluation of a 32-QAM 1-meter wireless link operating at $220-260 \mathrm{GHz}$ with a data-rate of $90 \mathrm{Gbps}$," in 2018 Asia-Pacific Microwave Conference (APMC), Nov 2018, pp. 723-725.
[14] P. Latzel, F. Pavanello, M. Billet, S. Bretin, A. Beck, M. Vanwolleghem, C. Coinon, X. Wallart, E. Peytavit, G. Ducournau, M. Zaknoune, and J. Lampin, "Generation of mw level in the 300-ghz band using resonantcavity-enhanced unitraveling carrier photodiodes," IEEE Transactions on Terahertz Science and Technology, vol. 7, no. 6, pp. 800-807, Nov 2017.

[15] A. Leuther, A. Tessmann, H. Massler, R. Losch, M. Schlechtweg, M. Mikulla, and O. Ambacher, "35 nm metamorphic HEMT MMIC technology," in Indium Phosphide and Related Materials, 2008. IPRM 2008. 20th International Conference on, May 2008, pp. 1-4.

[16] A. Tessmann, A. Leuther, S. Wagner, H. Massler, M. Kuri, H. . Stulz, M. Zink, M. Riessle, and T. Merkle, "A $300 \mathrm{GHz}$ low-noise amplifier S-MMIC for use in next-generation imaging and communication applications," in 2017 IEEE MTT-S International Microwave Symposium (IMS), June 2017, pp. 760-763.

[17] I. Dan, E. Rosello, P. Harati, S. Dilek, I. Kallfass, and S. Shiba, "Measurement of complex transfer function of analog transmit-receive frontends for terahertz wireless communications," in 2017 47th European Microwave Conference (EuMC), Oct 2017, pp. 1009-1012.

[18] F. Chang, K. Onohara, and T. Mizuochi, "Forward error correction for $100 \mathrm{G}$ transport networks," IEEE Communications Magazine, vol. 48 , no. 3, pp. S48-S55, March 2010. 